\title{
Recent Successes with a Meta-Logical Approach to Universal Logical Reasoning (Extended Abstract)
}

\author{
Christoph Benzmüller \\ University of Luxembourg, Luxembourg \& Freie Universität Berlin, Berlin, Germany
}

The quest for a most general framework supporting universal reasoning is very prominently represented in the works of Leibniz. He envisioned a scientia generalis founded on a characteristica universalis, that is, a most universal formal language in which all knowledge about the world and the sciences can be encoded. A quick study of the survey literature on logical formalisms suggests that quite the opposite to Leibniz' dream has become reality. Instead of a characteristica universalis, we are today facing a very rich and heterogenous zoo of different logical systems, and instead of converging towards a single superior logic, this logic zoo is further expanding, eventually even at accelerated pace. As a consequence, the unified vision of Leibniz seems farther away than ever before. However, there are also some promising initiatives to counteract these diverging developments. Attempts at unifying approaches to logic include categorial logic algebraic logic and coalgebraic logic.

My own research draws on another alternative at universal logical reasoning: the shallow semantical embeddings (SSE) approach. This approach has a very pragmatic motivation, foremost reuse of tools, simplicity and elegance. It utilises classical higherorder logic [22] as a unifying meta-logic in which the syntax and semantics of varying other logics can be explicitly modeled and flexibly combined (cf. [6] and the references therein). Off-the-shelf higher-order interactive and automated theorem provers [7] can then be employed to reason about and within the shallowly embedded logics.

Respective experiments have e.g. been conducted in metaphysics. An initial focus thereby has been on computer-supported assessments of modern variants of the ontological argument for the existence of God, where the SSE approach has been utilised in particular for automating variants of higher-order (multi-)modal logics [9].

In the course of these experiments (cf. [17|15|16]18 19|14] for details), my prover LEO-II [10] detected an previously unnoticed inconsistency in Kurt Gödel's [26] prominent variant of the ontological argument, while the slightly modified variant by Dana Scott [32] was verified in the interactive proof assistants Isabelle/HOL [30] and Coq [20]. Further modern variants of the argument have subsequently been studied with the approach, and theorem provers have even contributed to the clarification of an unsettled philosophical dispute [13].

Another, more ambitious study has focused on Ed Zalta's Principia Logico-Metaphysica (PLM) [38], which aims at a foundational logical theory for all of metaphysics and the sciences. This includes mathematics, and in this sense it is more ambitious than Russel's Principia Mathematica. The semantical embedding of PLM in HOL has been very challenging, since in addition to its size, its foundational theory is complicated: the PLM is based on hyperintensional higher-order modal logic S5 defined on top of a relational (as opposed to a functional) type theory that comes with restricted comprehension principles (the use of full comprehension in the PLM has been known to cause para- 
doxes and inconsistencies [31]). The PLM has meanwhile been successfully encoded in Isabelle/HOL by my student Daniel Kirchner [27]. As an unexpected highlight of this project, Kirchner, supported by the Isabelle/HOL system, detected an previously unnoticed issue: a deeply rooted and known paradox is reintroduced in PLM when the logic of complex terms is adjoined to PLM's specially-formulated comprehension principle for relations. Kirchner is now using the framework to support Zalta in fixing this issue.

Other logics, for which the SSE approach applies, and which are relevant for theoretical philosophy, include quantified conditional logics and multi-valued logic [5]4[35].

Motivated by the successful experiments on the ontological argument, and supported by my research group at Freie Universität (FU) Berlin, I have set-up a worldwide new lecture course on computational metaphysics [37], which has received FU Berlin's central teaching award in 2015/16. Student projects originating from this course have led to impressive new contributions (cf. [125]27]; further papers are submitted), including Kirchner's already mentioned embedding of the PLM in HOL, a computer-assisted reconstruction of an ontological argument by Leibniz and a verification of (main parts of) prominent textbooks by Fitting [23] and Boolos [21]. A key factor in the successful implementation of the course has been, that a single methodology and overall technique (the SSE approach) was used throughout, enabling the students to quickly adopt a wide range of different logic variants in short time within a single proof assistant (Isabelle/HOL). The course concept seems in fact well suited to significantly improve interdisciplinary, university level logic education.

Another interesting application area for the SSE approach is mathematics, where e.g. the proper treatment of partiality and undefinedness in computer-formalisations constitute unsettled challenges. Free logic [33|29] adapts classical logic in a way particularly suited for addressing them. Free logics have interesting applications, e.g. in natural language processing and as a logic of fiction. In mathematics, free logics are particularly suited in application domains such as category theory or projective geometry (e.g. morphism composition in category theory is a partial operation). In a collaboration with Dana Scott, I have shown that free logics can be elegantly embedded and automated in HOL [11]. Utilising this embedding, we have conducted an exemplary theory exploration study in category theory [12], in the course of which theorem provers have revealed a previously unnoticed technical flaw (constricted inconsistency resp. missing axioms) in a prominent category theory textbook [24].

The SSE approach is, of course, relevant also for artificial intelligence and computer science. For example, the knowledge and belief of intelligent agents can be modelled with epistemic and doxastic logics, which are directly amenable to the SSE approach, since they are just particular modal logics. To demonstrate this, prominent AI puzzles about knowledge and belief, including the well known wise men puzzle, have been successfully automated [3|36]. Moreover, the semantic web description logic ALC is just a reinvention of basic multi-modal logic $\mathrm{K}$ and, hence, the SSE approach is immediately applicable to it. Access control logics have applications e.g. in computer security; again the SSE approach applies [2]. Further ongoing work e.g. adresses intuitionistic modal logic [28] and predicate dynamic logic.

In summary, the SSE approach is the most widely applied universal logical reasoning approach to date. Note, however, the difference to Leibniz' original idea (and to 
various strands of related work). Instead of a single, universal logic formalism, the SSE approach supports many different competing object logics from the logic zoo. No ontological commitment is thus enforced at the object logic level (e.g. the approach well supports both classical and intuitionistic object logics, and can even combine them [8]). The concrete selection of (a range of) object logic candidates is typically determined by the specific requirements of the application at hand. Only at meta-level a single, unifying logic is provided, namely HOL (or any richer logic incorporating HOL). By unfolding the embeddings of the object logics, problem representations are uniformly mapped to HOL. This way Leibniz' vision is realised in an indirect way: universal logical reasoning is established (only) at the meta-level in HOL.

\section{References}

1. Bentert, M., Benzmüller, C., Streit, D., Woltzenlogel Paleo, B.: Analysis of an ontological proof proposed by Leibniz. In: Tandy, C. (ed.) Death and Anti-Death, Volume 14: Four Decades after Michael Polanyi, Three Centuries after G.W. Leibniz. Ria University Press (2016)

2. Benzmüller, C.: Automating access control logic in simple type theory with LEO-II. In: Gritzalis, D., López, J. (eds.) Emerging Challenges for Security, Privacy and Trust, 24th IFIP TC 11 International Information Security Conference, SEC 2009, Pafos, Cyprus, May 18-20, 2009. Proceedings. IFIP, vol. 297, pp. 387-398. Springer (2009)

3. Benzmüller, C.: Combining and automating classical and non-classical logics in classical higher-order logic. Annals of Mathematics and Artificial Intelligence (Special issue Computational logics in Multi-agent Systems (CLIMA XI)) 62(1-2), 103-128 (2011)

4. Benzmüller, C.: Automating quantified conditional logics in HOL. In: Rossi, F. (ed.) IJCAI 2013. pp. 746-753. AAAI Press (2013)

5. Benzmüller, C.: Cut-elimination for quantified conditional logic. Journal of Philosophical Logic 46(3), 333-353 (2017)

6. Benzmüller, C.: Universal reasoning, rational argumentation and human-machine interaction. arXiv, http://arxiv.org/abs/1703.09620(2017)

7. Benzmüller, C., Miller, D.: Automation of higher-order logic. In: Gabbay, D.M., Siekmann, J.H., Woods, J. (eds.) Handbook of the History of Logic, Volume 9 - Computational Logic, pp. 215-254. North Holland, Elsevier (2014)

8. Benzmüller, C., Paulson, L.: Multimodal and intuitionistic logics in simple type theory. The Logic Journal of the IGPL 18(6), 881-892 (2010)

9. Benzmüller, C., Paulson, L.: Quantified multimodal logics in simple type theory. Logica Universalis (Special Issue on Multimodal Logics) 7(1), 7-20 (2013)

10. Benzmüller, C., Paulson, L.C., Sultana, N., Theiß, F.: The higher-order prover LEO-II. Journal of Automated Reasoning 55(4), 389-404 (2015)

11. Benzmüller, C., Scott, D.: Automating free logic in Isabelle/HOL. In: Greuel, G.M., Koch, T., Paule, P., Sommese, A. (eds.) Mathematical Software - ICMS 2016, 5th International Congress, Proceedings. LNCS, vol. 9725, pp. 43-50. Springer, Berlin, Germany (2016)

12. Benzmüller, C., Scott, D.S.: Axiomatizing category theory in free logic. arXiv, http:// arxiv.org/abs/1609.01493(2016)

13. Benzmüller, C., Weber, L., Woltzenlogel Paleo, B.: Computer-assisted analysis of the Anderson-Hájek controversy. Logica Universalis 11(1), 139-151 (2017)

14. Benzmüller, C., Woltzenlogel Paleo, B.: Gödel's God in Isabelle/HOL. Archive of Formal Proofs (2013), (Formally verified) 
15. Benzmüller, C., Woltzenlogel Paleo, B.: Automating Gödel's ontological proof of God's existence with higher-order automated theorem provers. In: Schaub, T., Friedrich, G., O'Sullivan, B. (eds.) ECAI 2014. Frontiers in Artificial Intelligence and Applications, vol. 263, pp. 93 - 98. IOS Press (2014)

16. Benzmüller, C., Woltzenlogel Paleo, B.: Interacting with modal logics in the Coq proof assistant. In: Beklemishev, L.D., Musatov, D.V. (eds.) CSR 2015. LNCS, vol. 9139, pp. 398-411. Springer (2015)

17. Benzmüller, C., Woltzenlogel Paleo, B.: The inconsistency in Gödel's ontological argument: A success story for AI in metaphysics. In: Kambhampati, S. (ed.) IJCAI 2016. vol. 1-3, pp. 936-942. AAAI Press (2016)

18. Benzmüller, C., Woltzenlogel Paleo, B.: The modal collapse as a collapse of the modal square of opposition. In: Béziau, J.Y., Basti, G. (eds.) The Square of Opposition: A Cornerstone of Thought, pp. 307-313. Studies in Universal Logic, Springer (2016)

19. Benzmüller, C., Woltzenlogel Paleo, B.: Experiments in Computational Metaphysics: Gödel's proof of God's existence. Savijnanam: scientific exploration for a spiritual paradigm. Journal of the Bhaktivedanta Institute 9, 43-57 (2017)

20. Bertot, Y., Casteran, P.: Interactive Theorem Proving and Program Development. Springer (2004)

21. Boolos, G.: The Logic of Provability. Cambridge University Press (1993)

22. Church, A.: A formulation of the simple theory of types. J. Symbolic Logic 5, 56-68 (1940)

23. Fitting, M.: Types, Tableaus, and Gödel's God. Kluwer (2002)

24. Freyd, P.J., Scedrov, A.: Categories, Allegories. North Holland (1990)

25. Fuenmayor, D., Benzmüller, C.: Automating emendations of the ontological argument in intensional higher-order modal logic. In: KI 2017. LNAI, Springer (2017)

26. Gödel, K.: Appx. A: Notes in Kurt Gödel's Hand, pp. 144-145. In: Sobel [34] (1970)

27. Kirchner, D.: Representation and partial automation of the principia logico-metaphysica in isabelle/hol. Archive of Formal Proofs (2017), formally verified with Isabelle/HOL

28. Lachnitt, H.: Systematic verification of the intuitionistic modal logic cube in isabelle/hol. Bachelor Thesis at the Freie Universität Berlin, Institut für Informatik (2017)

29. Lambert, K.: Free Logic. Selected Essays. Cambridge University Press (2012)

30. Nipkow, T., Paulson, L., Wenzel, M.: Isabelle/HOL: A Proof Assistant for Higher-Order Logic. No. 2283 in LNCS, Springer (2002)

31. Oppenheimer, P.E., Zalta, E.N.: Relations versus functions at the foundations of logic: Typetheoretic considerations. J. Log. Comput. 21(2), 351-374 (2011)

32. Scott, D.: Appx. B: Notes in Dana Scott's Hand, pp. 145-146. In: Sobel [34] (1972)

33. Scott, D.: Existence and description in formal logic. In: Schoenman, R. (ed.) Bertrand Russell: Philosopher of the Century, pp. 181-200. George Allen \& Unwin, London (1967), (Reprinted with additions in: Philosophical Application of Free Logic, edited by K. Lambert. Oxford Universitry Press, 1991, pp. 28 - 48)

34. Sobel, J.: Logic and Theism. Cambridge U. Press (2004)

35. Steen, A., Benzmüller, C.: Sweet SIXTEEN: Automation via embedding into classical higher-order logic. Logic and Logical Philosophy 25, 535-554 (2016)

36. Steen, A., Wisniewski, M., Benzmüller, C.: Tutorial on reasoning in expressive non-classical logics with Isabelle/HOL. In: Benzüller, C., Rojas, R., Sutcliffe, G. (eds.) GCAI 2016. EPiC Series in Computing, vol. 41, pp. 1-10. EasyChair (2016)

37. Wisniewski, M., Steen, A., Benzmüller, C.: Einsatz von Theorembeweisern in der Lehre. In: Schwill, A., Lucke, U. (eds.) Hochschuldidaktik der Informatik: 7. Fachtagung des GIFachbereichs Informatik und Ausbildung/Didaktik der Informatik. Commentarii informaticae didacticae (CID), Universitätsverlag Potsdam, Potsdam, Germany (2016)

38. Zalta, E.N.: Principia logico-metaphysica (2016), draft version, preprint available at https://mally.stanford.edu/principia.pdf 